Supplement of Atmos. Meas. Tech., 14, 3657-3672, 2021

https://doi.org/10.5194/amt-14-3657-2021-supplement

(C) Author(s) 2021. CC BY 4.0 License.

(c) (i)

Atmospheric
Measurement
Techniques

Supplement of

\title{
A field intercomparison of three passive air samplers for gaseous mercury in ambient air
}

Attilio Naccarato et al.

Correspondence to: Attilio Naccarato (attilio.naccarato@iia.cnr.it) and Frank Wania (frank.wania@utoronto.ca)

The copyright of individual parts of the supplement might differ from the article licence. 
Table S1 Detailed sampler deployment plan. Dates refer to 2019.

\begin{tabular}{|c|c|c|c|c|c|c|c|c|c|c|c|c|c|c|}
\hline Site & Deployment & $1 w$ & $2 w$ & $3 w$ & $4 w$ & $5 w$ & $6 w$ & $7 w$ & $8 w$ & $9 w$ & $10 w$ & $11 w$ & $12 w$ & Sampling start and stop \\
\hline \multirow{11}{*}{ Rende } & $1^{\text {st }} 2$-week & & & & & & & & & & & & & 5 Feb to $19 \mathrm{Feb}$ \\
\hline & $2^{\text {nd }} 2$-week & & & & & & & & & & & & & 19 Feb to 5 Mar \\
\hline & $3^{\text {rd }} 2$-week & & & & & & & & & & & & & 5 Mar to 19 Mar \\
\hline & $4^{\text {th }} 2$-week & & & & & & & & & & & & & $2 \mathrm{Apr}$ to $16 \mathrm{Apr}$ \\
\hline & $1^{\text {st }} 4$-week & & & & & & & & & & & & & 5 Feb to 5 Mar \\
\hline & $2^{\text {nd }} 4$-week & & & & & & & & & & & & & 5 Mar to $2 \mathrm{Apr}$ \\
\hline & $3^{\text {rd }} 4$-week & & & & & & & & & & & & & $2 \mathrm{Apr}$ to $30 \mathrm{Apr}$ \\
\hline & $1^{\text {st }} 6$-week & & & & & & & & & & & & & 5 Feb to 19 Mar \\
\hline & $2^{\text {nd }} 6$-week & & & & & & & & & & & & & 19 Mar to $30 \mathrm{Apr}$ \\
\hline & $1^{\text {st }} 8$-week & & & & & & & & & & & & & $5 \mathrm{Feb}$ to $2 \mathrm{Apr}$ \\
\hline & $1^{\text {st }} 12$-week & & & & & & & & & & & & & 5 Feb to $30 \mathrm{Apr}$ \\
\hline \multirow{11}{*}{ Toronto } & $1^{\text {st }} 2$-week & & & & & & & & & & & & & 5 Feb to 19 Feb \\
\hline & $2^{\text {nd }} 2$-week & & & & & & & & & & & & & 19 Feb to 5 Mar \\
\hline & $3^{\text {rd }} 2$-week & & & & & & & & & & & & & 5 Mar to 20 Mar \\
\hline & $4^{\text {th }} 2$-week & & & & & & & & & & & & & $2 \mathrm{Apr}$ to $16 \mathrm{Apr}$ \\
\hline & $1^{\text {st }} 4$-week & & & & & & & & & & & & & 5 Feb to 5 Mar \\
\hline & $2^{\text {nd }} 4$-week & & & & & & & & & & & & & 5 Mar to $2 \mathrm{Apr}$ \\
\hline & $3^{\text {rd }} 4$-week & & & & & & & & & & & & & $2 \mathrm{Apr}$ to $30 \mathrm{Apr}$ \\
\hline & $1^{\text {st }} 6$-week & & & & & & & & & & & & & 5 Feb to $20 \mathrm{Mar}$ \\
\hline & $2^{\text {nd }} 6$-week & & & & & & & & & & & & & 20 Mar to $30 \mathrm{Apr}$ \\
\hline & $1^{\text {st }} 8$-week & & & & & & & & & & & & & 5 Feb to $2 \mathrm{Apr}$ \\
\hline & $1^{\text {st }} 12$-week & & & & & & & & & & & & & $5 \mathrm{Feb}$ to $30 \mathrm{Apr}$ \\
\hline
\end{tabular}


Figure S2 Photographs of the deployment set up in Toronto (left) and Rende (right).
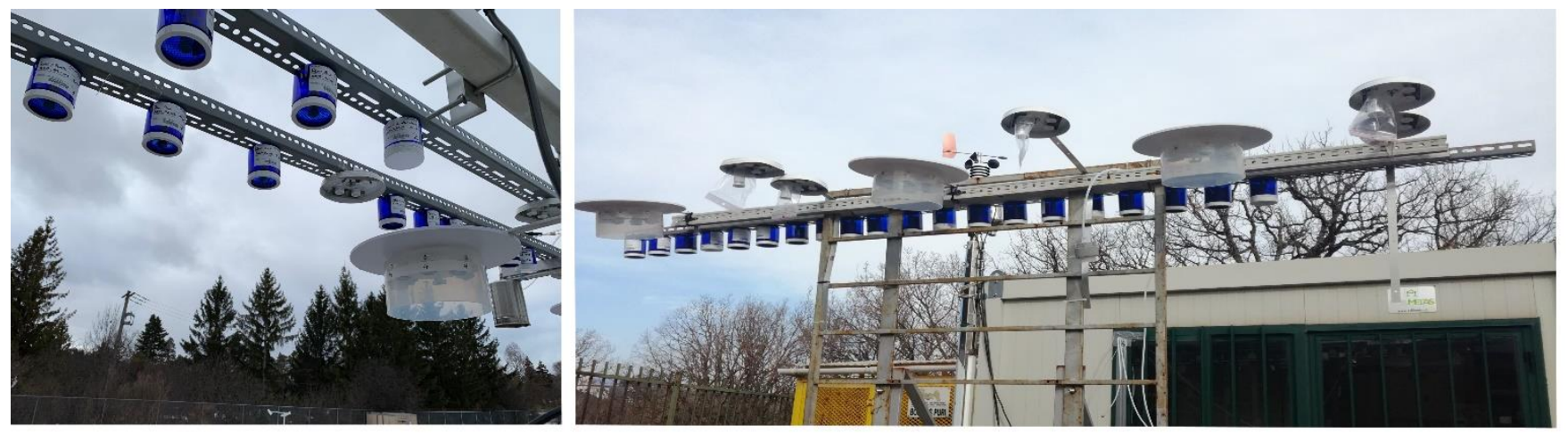
Figure S2. Concentration of gaseous mercury (5 minutes resolution) at Rende and Toronto using active instrumentation. Gaps in sampling data are due to automatic calibrations, maintenance operations and power failure.

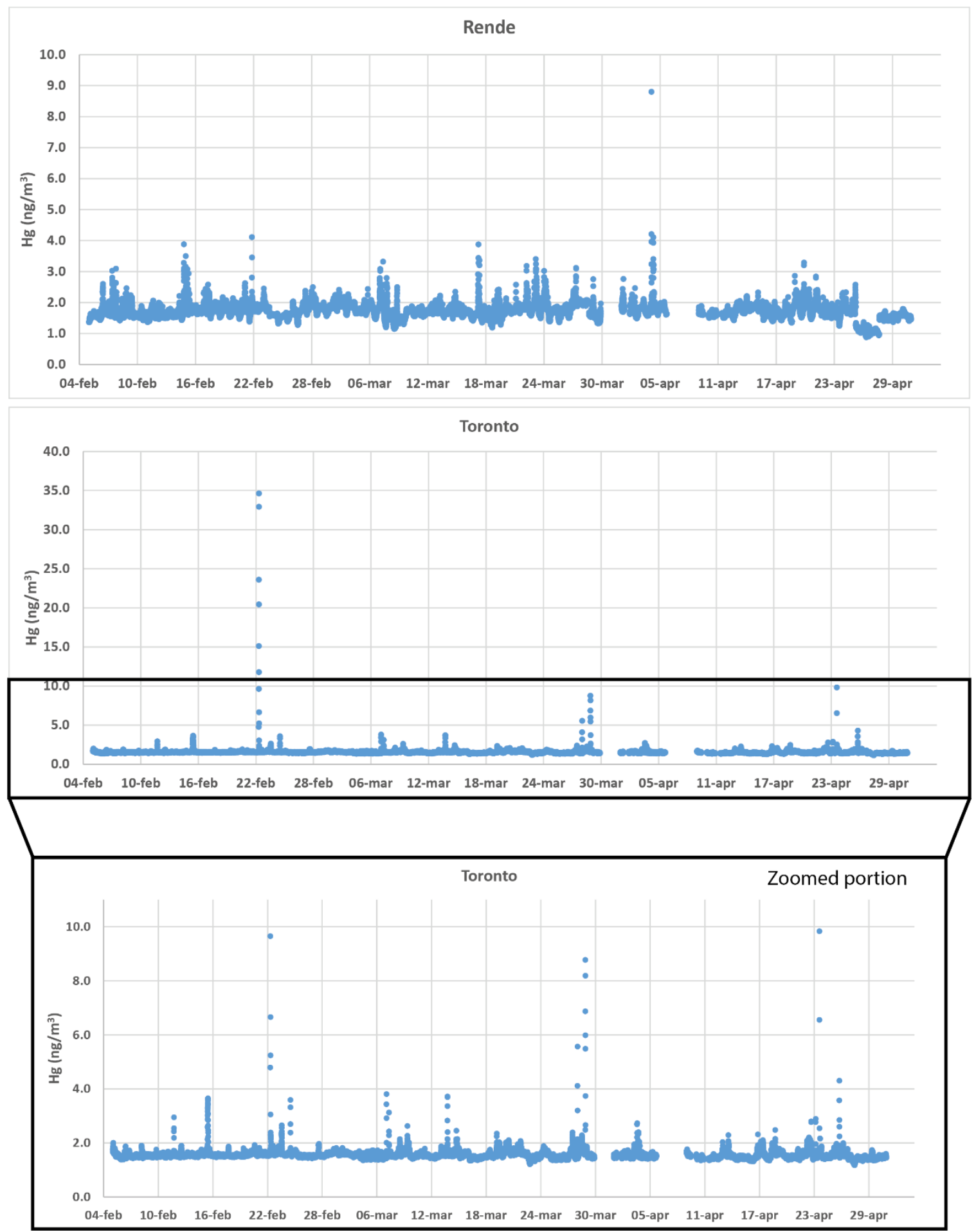


Table S2. Mean, standard deviation (SD) and percentage relative standard deviation (\%RSD) of mercury levels (nanograms) in field blanks for MerPAS ${ }^{\circledR}$, IVL-PAS, and CNR-PAS. The values are reported for each deployment period at both exposure sites. In Toronto the field blanks of the IVL-PAS were not deployed alongside the exposed samplers. $n$ designates the number of field blanks of a certain deployment length. The average values for each location (in bold font) are used for the blank correction of the amount of mercury measured in exposed passive air samplers.

\begin{tabular}{|c|c|c|cc|cc|cc|}
\hline & & \multicolumn{2}{|c|}{ MerPAS $^{\circledR}$} & \multicolumn{2}{c|}{ IVL-PAS } & \multicolumn{2}{c|}{ CNR-PAS } \\
& Length & $\boldsymbol{n}$ & Mean \pm SD & RSD & Mean \pm SD & RSD & Mean \pm SD & RSD \\
\hline \multirow{5}{*}{ Rende } & 2 weeks & 4 & $0.228 \pm 0.012$ & $5 \%$ & $0.495 \pm 0.079^{\mathrm{b}}$ & $16 \%$ & $0.144 \pm 0.014$ & $10 \%$ \\
& 4 weeks & 3 & $0.244 \pm 0.014$ & $6 \%$ & $0.482 \pm 0.110^{\mathrm{c}}$ & $23 \%$ & $0.155 \pm 0.029$ & $19 \%$ \\
& 6 weeks & 2 & $0.263^{\mathrm{a}}$ & - & $0.576 \pm 0.072$ & $12 \%$ & $0.151 \pm 0.014$ & $9 \%$ \\
& 8 weeks & 1 & 0.213 & - & 0.440 & - & 0.175 & - \\
& 12 weeks & 1 & 0.315 & - & 0.390 & - & 0.174 & - \\
\hline & all & $\mathbf{1 1}$ & $\mathbf{0 . 2 2 9} \pm \mathbf{0 . 0 5 5}$ & $\mathbf{2 4 \%}$ & $\mathbf{0 . 4 9 0} \pm \mathbf{0 . 0 9 0}$ & $\mathbf{1 8 \%}$ & $\mathbf{0 . 1 5 4} \pm \mathbf{0 . 0 1 9}$ & $\mathbf{1 2 \%}$ \\
\hline & 2 weeks & 4 & $0.200 \pm 0.041$ & $21 \%$ & - & - & $0.136 \pm 0.052$ & $38 \%$ \\
& 4 weeks & 3 & $0.258 \pm 0.040$ & $16 \%$ & - & - & $0.275 \pm 0.053$ & $19 \%$ \\
& 6 weeks & 2 & $0.240 \pm 0.068$ & $28 \%$ & - & - & $0.231 \pm 0.023$ & $10 \%$ \\
& 8 weeks & 1 & 0.243 & - & - & - & 0.211 & - \\
& 12 weeks & 1 & 0.322 & - & - & - & 0.195 & - \\
\hline
\end{tabular}


Table S3. Method detection limit (MDL), practical quantification limit (PQL) and limits of detection (LOD) and quantification (LOQ) for mercury for the three passive air samplers used in this study. Because the field blank levels were different in the two deployment locations, these method performance metrics are given for Rende and Toronto separately.

\begin{tabular}{|c|c|c|c|c|c|c|c|}
\hline & \multirow{2}{*}{$\begin{array}{c}\text { Length of } \\
\text { Deployment } \\
\text { (days) }\end{array}$} & \multicolumn{2}{|c|}{ MerPAS } & \multicolumn{2}{c|}{ IVL-PAS } & \multicolumn{2}{c|}{ CNR-PAS } \\
\cline { 3 - 8 } & & Rende & Toronto & Rende & Toronto & Rende & Toronto \\
\hline MDL (ng) & & 0.17 & 0.16 & 0.27 & 0.23 & 0.06 & 0.21 \\
PQL (ng) & & 0.55 & 0.52 & 0.90 & 0.77 & 0.19 & 0.70 \\
\hline \multirow{3}{*}{ LOD $\left(\mathrm{ng} \mathrm{m}^{-3}\right)$} & 14 & 0.11 & 0.10 & 0.64 & 0.55 & 0.28 & 1.02 \\
& 28 & 0.05 & 0.05 & 0.32 & 0.27 & 0.14 & 0.51 \\
& 42 & 0.04 & 0.03 & 0.21 & 0.18 & 0.09 & 0.34 \\
& 56 & 0.03 & 0.03 & 0.16 & 0.14 & 0.07 & 0.26 \\
& 84 & 0.02 & 0.02 & 0.11 & 0.09 & 0.05 & 0.17 \\
\hline \multirow{4}{*}{ LOQ } & 14 & 0.35 & 0.33 & 2.14 & 1.82 & 0.92 & 3.40 \\
$\left(\mathrm{ng} \mathrm{m}^{-3}\right)$ & 28 & 0.18 & 0.17 & 1.07 & 0.91 & 0.46 & 1.70 \\
& 42 & 0.12 & 0.11 & 0.71 & 0.61 & 0.31 & 1.13 \\
& 56 & 0.09 & 0.08 & 0.54 & 0.46 & 0.23 & 0.85 \\
& 84 & 0.06 & 0.06 & 0.36 & 0.30 & 0.15 & 0.57 \\
\hline
\end{tabular}


Table S4. Field blanks expressed as percentage of the amount of mercury in exposed samplers. The average of triplicate deployments is given in the top panel. The bottom panel shows the percentages averaged over deployment length.

\begin{tabular}{|c|c|c|c|c|c|c|}
\hline \multirow[b]{2}{*}{ Deployment } & \multicolumn{2}{|c|}{$\operatorname{MerPAS}^{\circledR}$} & \multicolumn{2}{|c|}{ IVL-PAS } & \multicolumn{2}{|c|}{ CNR-PAS } \\
\hline & Rende & Toronto & Rende & Toronto & Rende & Toronto \\
\hline $1^{\text {st }} 2$-week & $8 \%$ & $8 \%$ & $43 \%$ & $37 \%$ & $31 \%$ & $42 \%$ \\
\hline $2^{\text {nd }} 2$-week & $8 \%$ & $8 \%$ & $43 \%$ & $36 \%$ & $31 \%$ & $32 \%$ \\
\hline $3^{\text {rd }} 2$-week & $8 \%$ & $8 \%$ & $40 \%$ & $29 \%$ & $30 \%$ & $28 \%$ \\
\hline $4^{\text {th }} 2$-week & $8 \%$ & $9 \%$ & $43 \%$ & $36 \%$ & $30 \%$ & $47 \%$ \\
\hline $1^{\text {st }} 4$-week & $4 \%$ & $4 \%$ & $28 \%$ & $23 \%$ & $18 \%$ & $37 \%$ \\
\hline $2^{\text {nd }} 4$-week & $4 \%$ & $4 \%$ & $23 \%$ & $21 \%$ & $16 \%$ & $32 \%$ \\
\hline $3^{\text {rd }} 4$-week & $4 \%$ & $4 \%$ & $27 \%$ & $23 \%$ & $17 \%$ & $33 \%$ \\
\hline $1^{\text {st }} 6$-week & $3 \%$ & $3 \%$ & $21 \%$ & $17 \%$ & $13 \%$ & $18 \%$ \\
\hline $2^{\text {nd }} 6$-week & $3 \%$ & $3 \%$ & $21 \%$ & $17 \%$ & $13 \%$ & $19 \%$ \\
\hline $1^{\text {st }} 8$-week & $2 \%$ & $2 \%$ & $16 \%$ & $15 \%$ & $11 \%$ & $13 \%$ \\
\hline $1^{\text {st }} 12$-week & $1 \%$ & $1 \%$ & $10 \%$ & $10 \%$ & $7 \%$ & $11 \%$ \\
\hline 2-week $(n=8)$ & $7.9 \pm$ & $0.4 \%$ & 38 & $5 \%$ & 34 & $7 \%$ \\
\hline 4-week $(n=6)$ & $4.2 \pm$ & $0.1 \%$ & 24 & $3 \%$ & 26 & $9 \%$ \\
\hline 6-week $(n=4)$ & $2.7 \pm$ & $0.1 \%$ & 19 & $2 \%$ & & $3 \%$ \\
\hline 8 -week $(n=2)$ & $2.1 \pm$ & $0.1 \%$ & 15.2 & $0.7 \%$ & 12.0 & $1.2 \%$ \\
\hline 12-week $(n=2)$ & $1.38 \pm$ & $0.01 \%$ & 10.0 & $0.2 \%$ & & $2 \%$ \\
\hline
\end{tabular}


Table S5. Mean, standard deviation (SD) and percentage relative standard deviation (\%RSD) of the amounts of mercury quantified in the deployed passive air samplers (prior to blank correction) for each of the 22 deployments. All values are for triplicates unless otherwise noted.

\begin{tabular}{|c|c|c|c|c|c|c|c|}
\hline \multirow[b]{2}{*}{ Location } & \multirow[b]{2}{*}{ Deployment } & \multicolumn{2}{|c|}{$\operatorname{MerPAS}^{\circledR}$} & \multicolumn{2}{|c|}{ IVL-PAS } & \multicolumn{2}{|c|}{ CNR-PAS } \\
\hline & & $\begin{array}{c}\text { Mean } \pm \text { SD } \\
(n g)\end{array}$ & $\begin{array}{c}\text { RSD } \\
(\%)\end{array}$ & $\begin{array}{c}\text { Mean } \pm \text { SD } \\
(n g)\end{array}$ & $\begin{array}{c}\text { RSD } \\
(\%)\end{array}$ & $\begin{array}{c}\text { Mean } \pm \text { SD } \\
(\text { ng) }\end{array}$ & $\begin{array}{l}\text { RSD } \\
(\%)\end{array}$ \\
\hline \multirow{11}{*}{ Rende } & $1^{\text {st }} 2$-week & $2.99 \pm 0.11$ & 4 & $1.15 \pm 0.09$ & 8 & $0.50 \pm 0.02$ & 3 \\
\hline & $2^{\text {nd }} 2$-week & $3.00 \pm 0.06$ & 2 & $1.15 \pm 0.09$ & 8 & $0.50 \pm 0.02$ & 4 \\
\hline & $3^{\text {rd }} 2$-week & $2.92 \pm 0.27$ & 9 & $1.21 \pm 0.09$ & 7 & $0.51 \pm 0.03$ & 5 \\
\hline & $4^{\text {th }} 2$-week & $3.02 \pm 0.11$ & 4 & $1.15 \pm 0.09$ & 8 & $0.52 \pm 0.01$ & 3 \\
\hline & $1^{\text {st }} 4$-week & $5.72 \pm 0.29$ & 5 & $1.74 \pm 0.15$ & 8 & $0.84 \pm 0.12$ & 14 \\
\hline & $2^{\text {nd }} 4$-week & $5.72 \pm 0.14$ & 2 & $2.10 \pm 0.62$ & 29 & $0.96 \pm 0.02$ & 2 \\
\hline & $3^{\text {rd }} 4$-week & $5.44 \pm 0.18$ & 3 & $1.82 \pm 0.32$ & 17 & $0.89 \pm 0.03$ & 3 \\
\hline & $1^{\text {st }} 6$-week & $8.71 \pm 0.23^{\mathrm{a}}$ & 3 & $2.39 \pm 0.26$ & 11 & $1.15 \pm 0.11$ & 9 \\
\hline & $2^{\text {nd }} 6$-week & $8.69 \pm 0.11$ & 1 & $2.38 \pm 0.24$ & 10 & $1.20 \pm 0.05$ & 4 \\
\hline & $1^{\text {st }} 8$-week & $11.41 \pm 0.43$ & 4 & $3.12 \pm 0.25$ & 8 & $1.38 \pm 0.15$ & 11 \\
\hline & $1^{\text {st }} 12$-week & $16.61 \pm 0.30$ & 2 & $4.80 \pm 0.33$ & 7 & $2.13 \pm 0.35^{\mathrm{a}}$ & 16 \\
\hline \multirow{11}{*}{ Toronto } & $1^{\text {st }} 2$-week & $2.84 \pm 0.14$ & 5 & $1.17 \pm 0.05$ & 4 & $0.48 \pm 0.04$ & 7 \\
\hline & $2^{\text {nd }} 2$-week & $3.12 \pm 0.11$ & 4 & $1.19^{b}$ & & $0.64 \pm 0.06$ & 10 \\
\hline & $3^{\text {rd }} 2$-week & $2.90 \pm 0.20$ & 7 & $1.49 \pm 0.20$ & 13 & $0.73 \pm 0.07$ & 10 \\
\hline & $4^{\text {th }} 2$-week & $2.80 \pm 0.10$ & 4 & $1.18 \pm 0.01^{\mathrm{a}}$ & 1 & $0.43 \pm 0.03$ & 6 \\
\hline & $1^{\text {st }} 4$-week & $5.50 \pm 0.05$ & 1 & $1.91 \pm 0.10$ & 5 & $0.55 \pm 0.01$ & 3 \\
\hline & $2^{\text {nd }} 4$-week & $5.64 \pm 0.30$ & 5 & $2.09 \pm 0.27$ & 13 & $0.63 \pm 0.01$ & 2 \\
\hline & $3^{\text {rd }} 4$-week & $5.67 \pm 0.14$ & 2 & $1.89 \pm 0.13$ & 7 & $0.62 \pm 0.03$ & 4 \\
\hline & $1^{\text {st }} 6$-week & $8.28 \pm 0.33$ & 4 & $2.52 \pm 0.21$ & 8 & $1.10 \pm 0.09$ & 9 \\
\hline & $2^{\text {nd }} 6$-week & $8.48 \pm 0.23$ & 3 & $2.54 \pm 0.32$ & 13 & $1.05 \pm 0.08$ & 7 \\
\hline & $1^{\text {st }} 8$-week & $11.12 \pm 0.15$ & 1 & $2.91 \pm 0.04$ & 1 & $1.59 \pm 0.26$ & 16 \\
\hline & $1^{\text {st }} 12$-week & $17.11 \pm 0.27$ & 2 & $4.36 \pm 0.11$ & 2 & $1.91 \pm 0.10^{\mathrm{a}}$ & 5 \\
\hline
\end{tabular}

${ }^{\mathrm{a}}$ duplicate only, ${ }^{\mathrm{b}}$ no replication 
Table S6. Mean, standard deviation (SD) and relative standard deviation (RSD) of the amount of mercury quantified in the deployed passive air samplers (after blank correction) for each of the 22 deployments. All values are for triplicates unless otherwise noted. Standard deviations are calculated by propagating the standard deviation of both the amounts in Table S4 and the field blank levels in Table S1.

\begin{tabular}{|c|c|c|c|c|c|c|c|}
\hline \multirow[b]{2}{*}{ Location } & \multirow[b]{2}{*}{ Deployment } & \multicolumn{2}{|c|}{$\operatorname{MerPAS}^{\circledR}$} & \multicolumn{2}{|c|}{ IVL-PAS } & \multicolumn{2}{|c|}{ CNR-PAS } \\
\hline & & $\begin{array}{c}\text { Mean } \pm \text { SD } \\
(\mathrm{ng})\end{array}$ & $\begin{array}{l}\text { RSD } \\
(\%)\end{array}$ & $\begin{array}{c}\text { Mean } \pm \text { SD } \\
(\mathrm{ng})\end{array}$ & $\begin{array}{c}\text { RSD } \\
(\%)\end{array}$ & $\begin{array}{c}\text { Mean } \pm \text { SD } \\
(\mathrm{ng})\end{array}$ & $\begin{array}{l}\text { RSD } \\
(\%)\end{array}$ \\
\hline \multirow{11}{*}{ Rende } & $1^{\text {st }} 2$-week & $2.76 \pm 0.12$ & 4 & $0.66 \pm 0.13$ & 19 & $0.34 \pm 0.02$ & 7 \\
\hline & $2^{\text {nd }} 2$-week & $2.77 \pm 0.08$ & 3 & $0.66 \pm 0.13$ & 20 & $0.35 \pm 0.03$ & 8 \\
\hline & $3^{\text {rd }} 2$-week & $2.69 \pm 0.28$ & 10 & $0.72 \pm 0.13$ & 18 & $0.36 \pm 0.03$ & 9 \\
\hline & $4^{\text {th }} 2$-week & $2.79 \pm 0.12$ & 4 & $0.66 \pm 0.13$ & 19 & $0.36 \pm 0.02$ & 7 \\
\hline & $1^{\text {st }} 4$-week & $5.49 \pm 0.30$ & 5 & $1.25 \pm 0.17$ & 14 & $0.69 \pm 0.12$ & 18 \\
\hline & $2^{\text {nd }} 4$-week & $5.49 \pm 0.15$ & 3 & $1.61 \pm 0.62$ & 39 & $0.81 \pm 0.03$ & 4 \\
\hline & $3^{\text {rd }} 4$-week & $5.21 \pm 0.19$ & 4 & $1.33 \pm 0.33$ & 25 & $0.73 \pm 0.04$ & 5 \\
\hline & $1^{\text {st }} 6$-week & $8.48 \pm 0.24^{\mathrm{a}}$ & 3 & $1.90 \pm 0.28$ & 15 & $1.00 \pm 0.11$ & 11 \\
\hline & $2^{\text {nd }} 6$-week & $8.46 \pm 0.12$ & 1 & $1.89 \pm 0.26$ & 14 & $1.05 \pm 0.05$ & 5 \\
\hline & $1^{\text {st }} 8$-week & $11.18 \pm 0.43$ & 4 & $2.63 \pm 0.27$ & 10 & $1.23 \pm 0.15$ & 12 \\
\hline & $1^{\text {st }} 12$-week & $16.38 \pm 0.31$ & 2 & $4.31 \pm 0.34$ & 8 & $1.97 \pm 0.35^{\mathrm{a}}$ & 18 \\
\hline \multirow{11}{*}{ Toronto } & $1^{\text {st }} 2$-week & $2.60 \pm 0.15$ & 6 & $0.74 \pm 0.09$ & 12 & $0.28 \pm 0.08$ & 28 \\
\hline & $2^{\text {nd }} 2$-week & $2.88 \pm 0.12$ & 4 & $0.76 \pm 0.08^{b}$ & 10 & $0.43 \pm 0.09$ & 21 \\
\hline & $3^{\text {rd }} 2$-week & $2.66 \pm 0.21$ & 8 & $1.06 \pm 0.21$ & 20 & $0.53 \pm 0.10$ & 19 \\
\hline & $4^{\text {th }} 2$-week & $2.56 \pm 0.11$ & 4 & $0.75 \pm 0.08^{\mathrm{a}}$ & 10 & $0.23 \pm 0.07$ & 33 \\
\hline & $1^{\text {st }} 4$-week & $5.26 \pm 0.07$ & 1 & $1.48 \pm 0.13$ & 9 & $0.35 \pm 0.07$ & 20 \\
\hline & $2^{\text {nd }} 4$-week & $5.40 \pm 0.33$ & 6 & $1.66 \pm 0.28$ & 17 & $0.43 \pm 0.07$ & 17 \\
\hline & $3^{\text {rd }} 4$-week & $5.43 \pm 0.15$ & 3 & $1.46 \pm 0.15$ & 10 & $0.42 \pm 0.08$ & 18 \\
\hline & $1^{\text {st }} 6$-week & $8.04 \pm 0.33$ & 4 & $2.09 \pm 0.22$ & 11 & $0.90 \pm 0.12$ & 13 \\
\hline & $2^{\text {nd }} 6$-week & $8.24 \pm 0.24$ & 3 & $2.11 \pm 0.33$ & 16 & $0.85 \pm 0.10$ & 12 \\
\hline & $1^{\text {st }} 8$-week & $10.88 \pm 0.16$ & 1 & $2.28 \pm 0.09$ & 3 & $1.38 \pm 0.27$ & 19 \\
\hline & $1^{\text {st }} 12$-week & $16.87 \pm 0.27$ & 2 & $3.93 \pm 0.13$ & 3 & $1.70 \pm 0.12^{\mathrm{a}}$ & 7 \\
\hline
\end{tabular}

${ }^{\mathrm{a}}$ duplicate only, ${ }^{\mathrm{b}}$ no replication 
Table S7. Average replicate precision (in \%) of the amount of mercury quantified in a PAS (mPAS) and of the blank-corrected amount of mercury in a PAS ( $\left.\mathrm{m}_{\mathrm{PAS}}-\mathrm{m}_{\mathrm{FB}}\right) . n$ designates the number of deployments of a certain type.

\begin{tabular}{|c|c|c|c|c|c|c|c|}
\hline \multirow{2}{*}{} & \multirow{2}{*}{$\boldsymbol{n}$} & \multicolumn{2}{|c|}{$\boldsymbol{M e r P A S}^{\circledR}$} & \multicolumn{2}{c|}{ IVL-PAS } & \multicolumn{2}{c|}{ CNR-PAS } \\
\cline { 3 - 8 } & & $\boldsymbol{m}_{\text {PAS }}$ & $\boldsymbol{m}_{\text {PAS- }} \boldsymbol{m}_{\mathbf{F B}}$ & $\boldsymbol{m}_{\text {PAS }}$ & $\boldsymbol{m}_{\text {PAS- }}$ FB & $\boldsymbol{m}_{\text {PAS }}$ & $\boldsymbol{m}_{\text {PAS- }} \boldsymbol{m}_{\text {FB }}$ \\
\hline 2-week deployments & 8 & 4.7 & 5.5 & 7.0 & 16 & 6.0 & 17 \\
4-week deployments & 6 & 3.3 & 3.6 & 13 & 19 & 4.8 & 14 \\
6-week deployments & 4 & 2.7 & 2.8 & 11 & 14 & 7.3 & 10 \\
8-week deployments & 2 & 2.6 & 2.7 & 4.7 & 6.8 & 13.4 & 16 \\
12-week deployments & 2 & 1.7 & 1.7 & 4.7 & 5.7 & 10.7 & 12 \\
Rende deployments & 11 & 3.5 & 4.0 & 11 & 18 & 6.9 & 9.3 \\
Toronto deployments & 11 & 3.4 & 3.8 & 6.8 & 11 & 7.2 & 19 \\
all deployments & 22 & 3.5 & 3.9 & 9.1 & 15 & 7.0 & 14 \\
\hline
\end{tabular}


Table S8. Mean, standard deviation (SD) and relative standard deviation (RSD) of the volumetric concentrations of mercury in air for each of the 22 deployments as derived by the three passive air samplers. All values refer to triplicates unless otherwise noted. Standard deviations are calculated by propagating the standard deviation of the amounts in Table S5 and the assumed uncertainty of the sampling rates.

\begin{tabular}{|c|c|c|c|c|c|c|c|}
\hline \multirow[b]{2}{*}{ Location } & \multirow[b]{2}{*}{ Deployment } & \multicolumn{2}{|c|}{$\operatorname{MerPAS}{ }^{\circledR}$} & \multicolumn{2}{|c|}{ IVL-PAS } & \multicolumn{2}{|c|}{ CNR-PAS } \\
\hline & & $\begin{array}{c}\text { Mean } \pm \text { SD } \\
\left(n g / m^{3}\right)\end{array}$ & $\begin{array}{l}\text { RSD } \\
(\%)\end{array}$ & $\begin{array}{c}\text { Mean } \pm \text { SD } \\
\left(n g / m^{3}\right)\end{array}$ & $\begin{array}{l}\text { RSD } \\
(\%)\end{array}$ & $\begin{array}{c}\text { Mean } \pm \text { SD } \\
\left(n g / m^{3}\right)\end{array}$ & $\begin{array}{l}\text { RSD } \\
(\%)\end{array}$ \\
\hline \multirow{11}{*}{ Rende } & $1^{\text {st }} 2$-week & $1.78 \pm 0.16$ & 9 & $1.66 \pm 0.20$ & 12 & $1.67 \pm 0.09$ & 5 \\
\hline & $2^{\text {nd }} 2$-week & $1.78 \pm 0.15$ & 9 & $1.66 \pm 0.21$ & 13 & $1.69 \pm 0.10$ & 6 \\
\hline & $3^{\text {rd }} 2$-week & $1.71 \pm 0.18$ & 10 & $1.84 \pm 0.19$ & 10 & $1.74 \pm 0.10$ & 5 \\
\hline & $4^{\text {th }}$ 2-week & $1.80 \pm 0.16$ & 9 & $1.67 \pm 0.20$ & 12 & $1.76 \pm 0.08$ & 4 \\
\hline & $1^{\text {st }} 4$-week & $1.77 \pm 0.16$ & 9 & $1.57 \pm 0.15$ & 10 & $1.68 \pm 0.18$ & 10 \\
\hline & $2^{\text {nd }} 4$-week & $1.77 \pm 0.15$ & 9 & $2.05 \pm 0.39$ & 19 & $1.96 \pm 0.06$ & 3 \\
\hline & $3^{\text {rd }} 4$-week & $1.68 \pm 0.15$ & 9 & $1.70 \pm 0.25$ & 15 & $1.78 \pm 0.07$ & 4 \\
\hline & $1^{\text {st }} 6$-week & $1.82 \pm 0.15^{\mathrm{a}}$ & 8 & $1.60 \pm 0.16$ & 10 & $1.61 \pm 0.12$ & 8 \\
\hline & $2^{\text {nd }} 6$-week & $1.82 \pm 0.15$ & 8 & $1.61 \pm 0.15$ & 9 & $1.69 \pm 0.07$ & 4 \\
\hline & $1^{\text {st }} 8$-week & $1.80 \pm 0.15$ & 9 & $1.67 \pm 0.12$ & 7 & $1.49 \pm 0.13$ & 9 \\
\hline & $1^{\text {st }} 12$-week & $1.76 \pm 0.15$ & 9 & $1.83 \pm 0.10$ & 5 & $1.60 \pm 0.18^{a}$ & 12 \\
\hline \multirow{11}{*}{ Toronto } & $1^{\text {st }} 2$-week & $1.65 \pm 0.16$ & 10 & $1.77 \pm 0.18$ & 10 & $1.35 \pm 0.28$ & 21 \\
\hline & $2^{\text {nd }} 2$-week & $1.87 \pm 0.16$ & 8 & $1.86 \pm 0.17^{b}$ & 9 & $2.12 \pm 0.22$ & 10 \\
\hline & $3^{\text {rd }} 2$-week & $1.60 \pm 0.17$ & 11 & $2.42 \pm 0.24$ & 10 & $2.40 \pm 0.20$ & 8 \\
\hline & $4^{\text {th }} 2$-week & $1.67 \pm 0.16$ & 9 & $1.90 \pm 0.17^{\mathrm{a}}$ & 9 & $1.11 \pm 0.33$ & 30 \\
\hline & $1^{\text {st }} 4$-week & $1.69 \pm 0.15$ & 9 & $1.79 \pm 0.16$ & 9 & $0.85 \pm 0.21$ & 25 \\
\hline & $2^{\text {nd }} 4$-week & $1.73 \pm 0.16$ & 9 & $2.03 \pm 0.22$ & 11 & $1.04 \pm 0.17$ & 17 \\
\hline & $3^{\text {rd }} 4$-week & $1.76 \pm 0.15$ & 9 & $1.84 \pm 0.17$ & 9 & $1.03 \pm 0.18$ & 18 \\
\hline & $1^{\text {st }} 6$-week & $1.68 \pm 0.16$ & 9 & $1.65 \pm 0.17$ & 10 & $1.42 \pm 0.14$ & 10 \\
\hline & $2^{\text {nd }} 6$-week & $1.82 \pm 0.15$ & 8 & $1.80 \pm 0.20$ & 11 & $1.41 \pm 0.13$ & 9 \\
\hline & $1^{\text {st }} 8$-week & $1.74 \pm 0.15$ & 9 & $1.51 \pm 0.13$ & 9 & $1.67 \pm 0.20$ & 12 \\
\hline & $1^{\text {st }} 12$-week & $1.81 \pm 0.15$ & 8 & $1.61 \pm 0.13$ & 8 & $1.38 \pm 0.08^{\mathrm{a}}$ & 6 \\
\hline
\end{tabular}

${ }^{a}$ duplicate only, ${ }^{\mathrm{b}}$ no replication 
Table S9. Variance decomposition analysis partitioning variability in the percent concentration differences between Tekran ${ }^{\circledR}$ and PASs, across four nested levels. Factors explaining the largest proportion of the variation are highlighted in bold.

\begin{tabular}{|c|c|}
\hline Variable & Proportion explained \\
\hline Site & $\mathbf{0 . 4 6 7 8}$ \\
\hline Period & 0.0000 \\
\hline PAS ID & $\mathbf{0 . 4 8 3 4}$ \\
\hline Tekran ID & 0.0474 \\
\hline Unexplained & 0.0015 \\
\hline
\end{tabular}

Table S10. Results of a mixed effects model predicting percent concentration differences between Tekran and PASs. Significant terms are highlighted in bold, and the model was fit while accounting for deployment time and location as random effects (where these random effects exerted a significant effect on concentration differences, $\left.\chi^{2}=8.7, p=0.003\right)$.

\begin{tabular}{|c|c|c|c|c|c|c|}
\hline Term & Sum Sq. & Mean Sq. & Num D.F. & Den. D.F. & $\boldsymbol{F}$ value & $\boldsymbol{p}$ value \\
\hline PAS ID & $\mathbf{1 9 0 3 . 6}$ & $\mathbf{9 5 1 . 8}$ & $\mathbf{2}$ & $\mathbf{8 3}$ & $\mathbf{5 . 3 7}$ & $\mathbf{0 . 0 0 6}$ \\
\hline Site & $\mathbf{5 0 3 6 . 6}$ & $\mathbf{5 0 3 6 . 6}$ & $\mathbf{1}$ & $\mathbf{8 3}$ & $\mathbf{2 8 . 4 3}$ & $<\mathbf{0 . 0 0 1}$ \\
\hline PAS ID*Site & 838.6 & 419.3 & 2 & 83 & 2.37 & 0.100 \\
\hline
\end{tabular}


Table S11. Statistical comparison of least square mean differences in concentrations, across different passive sampler models, sites, and all passive air sampler (PAS) model-by-site combinations. Comparisons here are based on a linear mixed effects model (presented in Table 2) predicting concentration differences as a function of sampler, site, and a sampler-by-site interaction, while accounting for sampling location and time as random effects. Significant differences are highlighted in bold.

\begin{tabular}{|c|c|c|c|c|c|c|c|}
\hline $\begin{array}{c}\text { Factors } \\
\text { compared }\end{array}$ & PAS 1 & Site 1 & PAS 2 & Site 2 & $\begin{array}{c}\text { Estimated } \\
\text { difference } \\
(\%)\end{array}$ & $t$ value & $p$ value \\
\hline PAS & CNR-PAS & - & $\operatorname{MerPAS}{ }^{\circledR}$ & - & 5.5 & 1.59 & 0.116 \\
\hline PAS & CNR-PAS & - & $\operatorname{MerPAS}^{\circledR}$ & - & 11.4 & 3.28 & 0.002 \\
\hline PAS & IVL-PAS & - & $\operatorname{MerPAS}^{\circledR}$ & - & 5.9 & 1.69 & 0.095 \\
\hline Sites & - & Rende & & Toronto & -15.1 & -5.33 & $<0.001$ \\
\hline PAS-by-Sites & CNR-PAS & Rende & IVL-PAS & Rende & -0.1 & -0.01 & 0.992 \\
\hline PAS-by-Sites & CNR-PAS & Rende & $\operatorname{MerPAS}{ }^{\circledR}$ & Rende & 4.2 & 0.74 & 0.464 \\
\hline PAS-by-Sites & CNR-PAS & Rende & CNR-PAS & Toronto & -23.7 & -4.81 & $<0.001$ \\
\hline PAS-by-Sites & CNR-PAS & Rende & IVL-PAS & Toronto & -12.6 & -2.56 & 0.012 \\
\hline PAS-by-Sites & CNR-PAS & Rende & $\operatorname{MerPAS}^{\circledR}$ & Toronto & -5.1 & -1.03 & 0.307 \\
\hline PAS-by-Sites & IVL-PAS & Rende & $\operatorname{MerPAS}^{\circledR}$ & Rende & 4.2 & 0.75 & 0.458 \\
\hline PAS-by-Sites & IVL-PAS & Rende & CNR-PAS & Toronto & -23.6 & -4.8 & $<0.001$ \\
\hline PAS-by-Sites & IVL-PAS & Rende & IVL-PAS & Toronto & -12.5 & -2.54 & 0.013 \\
\hline PAS-by-Sites & IVL-PAS & Rende & $\operatorname{MerPAS}^{\circledR}$ & Toronto & -5 & -1.02 & 0.312 \\
\hline PAS-by-Sites & MerPAS & Rende & CNR-PAS & Toronto & -27.8 & -5.66 & $<0.001$ \\
\hline PAS-by-Sites & MerPAS & Rende & IVL-PAS & Toronto & -16.7 & -3.41 & 0.001 \\
\hline PAS-by-Sites & MerPAS & Rende & $\operatorname{MerPAS}^{\circledR}$ & Toronto & -9.2 & -1.88 & 0.064 \\
\hline PAS-by-Sites & CNR-PAS & Toronto & IVL-PAS & Toronto & 11.1 & 2.76 & 0.007 \\
\hline PAS-by-Sites & CNR-PAS & Toronto & $\operatorname{MerPAS}^{\circledR}$ & Toronto & 18.6 & 4.64 & $<0.001$ \\
\hline PAS-by-Sites & IVL-PAS & Toronto & $\operatorname{MerPAS}^{\circledR}$ & Toronto & 7.5 & 1.87 & 0.065 \\
\hline
\end{tabular}

